\title{
Towards finite density QCD with Taylor expansions
}

\author{
F. Karsch \\ Physics Department, Brookhaven National Laboratory, Upton, NY 11973, USA \\ Fakultät für Physik, Universität Bielefeld, D-33615 Bielefeld, Germany \\ E-mail: karschephysik.uni-bielefeld.de, karsch@bnl.gov
}

\section{B.-J. Schaefer}

Institut für Physik, Karl-Franzens-Universität Graz, A-8010 Graz, Austria

Institut für Theoretische Physik, Justus-Liebig-Universität Gießen, D-35392 Gießen, Germany

E-mail: bernd-jochen.schaefer@uni-graz.at

\section{Wagner*}

Fakultät für Physik, Universität Bielefeld, D-33615 Bielefeld, Germany

E-mail: mwagner@physik.uni-bielefeld.de

\section{J. Wambach}

Institut für Kernphysik, TU Darmstadt, D-64289 Darmstadt, Germany

Gesellschaft für Schwerionenforschung GSI, D-64291 Darmstadt, Germany

E-Mail: jochen.wambach@physik.tu-darmstadt.de

\begin{abstract}
We analyze general convergence properties of the Taylor expansion of observables to finite chemical potential in the framework of an effective $2+1$ flavor Polyakov-quark-meson model. To compute the required higher order coefficients a novel technique based on algorithmic differentiation has been developed. Results for thermodynamic observables as well as the phase structure obtained through the series expansion up to 24th order are compared to the full model solution at finite chemical potential. The available higher order coefficients also allow for resummations, e.g. Padé series, which improve the convergence behavior. In view of our results we discuss the prospects for locating the QCD phase boundary and a possible critical endpoint with the Taylor expansion method.
\end{abstract}

XXIX International Symposium on Lattice Field Theory

July 10-16, 2011

Squaw Valley, Lake Tahoe, California

\footnotetext{
* Speaker.
} 


\section{Introduction}

The phase structure of strongly-interacting matter at non-vanishing temperature and densities and in particular the possible existence of a critical endpoint (CEP) is currently a very active frontier both theoretically and experimentally. Our present knowledge of the QCD phase diagram in the non-perturbative regime rests upon effective descriptions since first-principle approaches such as QCD lattice simulations mostly fail at large densities, for recent developments see e.g. [1]. Several extrapolation methods have been proposed to overcome the fermion sign problem. All of these approximations have their own problems and their reliability is still under investigations, see e.g. [2] for reviews. Therefore, it would stand to reason to combine the approximation schemes with QCDlike effective model calculations. A comparison of the analytical model results with the lattice calculations will shed light on their principal applicabilities. Here we focus on the Taylor expansion method [3] for small chemical potentials, see also [4].

It is based on an expansion of the pressure $p$ in powers of $x \equiv \mu / T$ around vanishing quark chemical potential $\mu$ :

$$
\frac{p(\mu / T)}{T^{4}}=\sum_{n=0}^{\infty} c_{n}(T)\left(\frac{\mu}{T}\right)^{n} \quad \text { with } \quad c_{n}(T)=\left.\frac{1}{n !} \frac{\partial^{n}\left(p(T, \mu) / T^{4}\right)}{\partial(\mu / T)^{n}}\right|_{\mu=0} .
$$

The relevant Taylor coefficients $c_{n}(T)$ can be calculated with standard techniques at $\mu=0$ [5]. The convergence of the series may be improved by a resummation based on a Padé approximation

$$
[L / M] \equiv R_{L, M}(x)=\frac{p(x)}{q(x)}=\frac{p_{0}+p_{1} x+\cdots+p_{L} x^{L}}{1+q_{1} x+\cdots+q_{M} x^{M}},
$$

where the Padé coefficients $p_{i}$ and $q_{j}$ can be extracted from the Taylor coefficients $c_{n}$ up to the order $L+M$. Hence, no further input is required for the application of a Padé resummation. By means of the Padé resummation often an extended convergence range and more stable results with fewer coefficients can be obtained, in particular in the presence of singularities.

\section{A model analysis}

As an effective QCD-like model framework we chose the $N_{f}=2+1$ flavor Polyakov quarkmeson (PQM) model which exhibits a chiral crossover at vanishing densities at $T_{\chi} \sim 206 \mathrm{MeV}$ and a CEP around $\left(T_{c}, \mu_{c}\right) \sim(185,167) \mathrm{MeV}$. Its thermodynamics at $\mu=0$ is in agreement with recent lattice simulations [6]. The PQM model Lagrangian consist of a quark-meson part

$$
\mathscr{L}_{\mathrm{PQM}}=\bar{q}\left(i \not D-h \phi_{5}\right) q+\mathscr{L}_{m}-\mathscr{U}(\Phi, \bar{\Phi}),
$$

where the $N_{f}$ quark fields $q$ are coupled via a Yukawa interaction $h$ to the scalar and pseudoscalar meson nonets $\phi$ and to the Polyakov loop via the covariant derivative. The purely mesonic contribution reads

$$
\begin{aligned}
\mathscr{L}_{m}= & \operatorname{Tr}\left(\partial_{\mu} \phi^{\dagger} \partial^{\mu} \phi\right)-m^{2} \operatorname{Tr}\left(\phi^{\dagger} \phi\right)-\lambda_{1}\left[\operatorname{Tr}\left(\phi^{\dagger} \phi\right)\right]^{2}-\lambda_{2} \operatorname{Tr}\left(\phi^{\dagger} \phi\right)^{2} \\
& +c\left(\operatorname{det}(\phi)+\operatorname{det}\left(\phi^{\dagger}\right)\right)+\operatorname{Tr}\left[H\left(\phi+\phi^{\dagger}\right)\right] .
\end{aligned}
$$




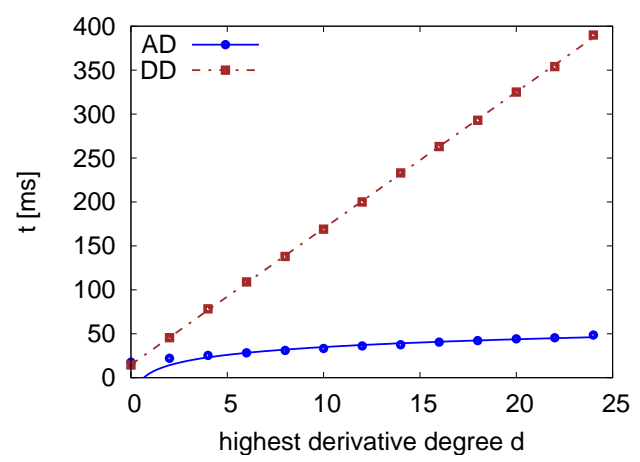

Figure 1: Runtime comparison for the DD and AD methods

The pure gauge sector is encoded (in the logarithmic version of) the Polyakov loop potential [7]

$$
\frac{\mathscr{U}_{\log }}{T^{4}}=-\frac{a(T)}{2} \bar{\Phi} \Phi+b(T) \ln \left[1-6 \bar{\Phi} \Phi+4\left(\Phi^{3}+\bar{\Phi}^{3}\right)-3(\bar{\Phi} \Phi)^{2}\right]
$$

whose parameters are fitted to lattice data. The matter back-reaction to the pure Yang-Mills system [9] is not considered in this work. The remaining model parameters are chosen to reproduce meson masses and decay constants in the vacuum [8]. The grand potential in mean-field approximation is obtained by integrating the quark loop which yields a function of the (non-strange and strange) chiral order parameters $\left(\sigma_{x}, \sigma_{y}\right)$ and the Polyakov-loop variables $(\Phi, \bar{\Phi})$

$$
\Omega=U\left(\sigma_{x}, \sigma_{y}\right)+\Omega_{\bar{q} q}\left(\sigma_{x}, \sigma_{y}, \Phi, \bar{\Phi}\right)+\mathscr{U}(\Phi, \bar{\Phi}) .
$$

Their temperature and chemical potential dependence is found by minimizing the grand potential

$$
\frac{\partial \Omega}{\partial \sigma_{x}}=\frac{\partial \Omega}{\partial \sigma_{y}}=\frac{\partial \Omega}{\partial \Phi}=\left.\frac{\partial \Omega}{\partial \bar{\Phi}}\right|_{\min }=0
$$

where $\min$ labels the global minimum of the potential. This introduces an only numerically invertible implicit $\mu$-dependence in the pressure $p(T, \mu)=-\left.\Omega(T, \mu)\right|_{\min }$.

An analytic evaluation of the Taylor coefficients is not only cumbersome due to the exponentially increasing number of terms but becomes finally impossible by the implicit $\mu$-dependence. Standard numerical derivate techniques such as divided differences (DD) fail at higher orders due to the increasing numerical errors. By means of the novel derivative technique, based on algorithmic differentiation $(\mathrm{AD})$, higher order derivatives can be calculated with extremely high precision, essentially limited only by machine precision. This works even in the case of only numerically solvable implicit dependences, such as in Eq. (2.4). Furthermore, also the performance of the AD technique is superior to the DD method as demonstrated in Fig. 1 where the time to calculate the $d$-th order derivatives is shown as a function of $d$. With the DD method one needs at least $d+1$ function evaluations for a $d$-th order derivatives and a linear $d$-dependence is obtained in contrast to the logarithmic dependence for the AD method, see [10] for further details. High-order Taylor coefficients calculated with the AD technique in the PQM model are given in [11]. The method is neither restricted to vanishing $\mu$ nor mean-field approximation and can be used to search for signals of the CEP in higher moments [14]. 


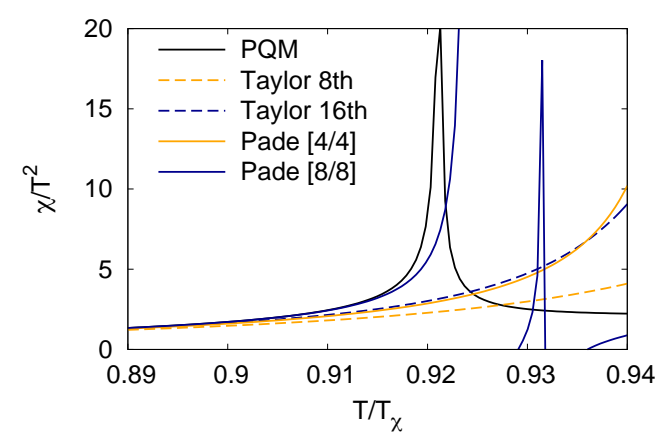

(a) $\mu / T=0.8$

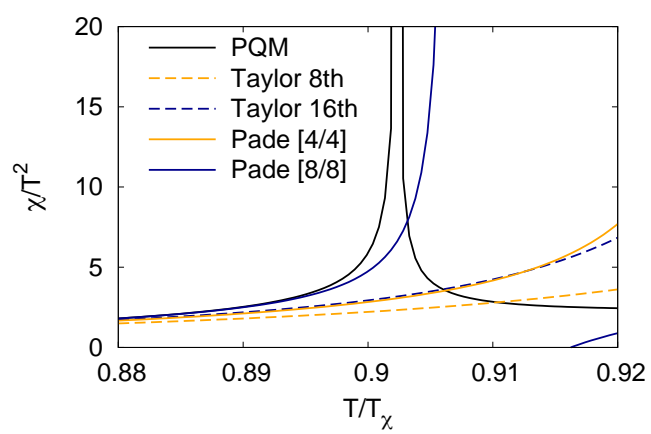

(b) $\mu / T=0.9$

Figure 2: Comparison of the quark number susceptibility in the mean-field model (PQM) with different orders of the extrapolation methods in the crossover region (left panel) and at the CEP (right panel).

\subsection{Thermodynamics}

The Taylor expansion can be applied straightforwardly to extrapolate thermodynamic quantities such as the quark-number susceptibility $\chi=\partial^{2} p /\left(\partial \mu^{2}\right)$ to finite densities. In Fig. 2 the scaled susceptibility $\chi / T^{2}$ calculated in the PQM model is compared to results obtained with the Taylor expansion and the Padé resummation. It diverges exactly at the CEP which is located for our chosen parameters at $\left(T_{c}, \mu_{c}\right) \sim(185,167) \mathrm{MeV}$, correspondingly $\mu_{c} / T_{c} \sim 0.9$ (right panel) and is finite otherwise. Both, the Taylor expansion and the Padé resummation cannot capture this divergence and yield finite values around $T_{c}$. For comparison the left panel shows $\chi$ in the crossover regime $(\mu / T=0.8)$. In the chirally broken phase, i.e., for temperatures smaller than the one of the $\chi$ peak, the agreement improves with increasing expansion orders. At larger temperatures $T \sim T_{\chi}$ the extrapolations exhibit some unphysical peaks which are not present in the model calculation. These peaks, which are also seen in extrapolations where lattice simulation coefficients have been used (see e.g. [12]), are solely an artifact of the extrapolation. However, an important observation is that in both cases the [4/4] Padé approximation yields nearly the same result as the 16-th order Taylor expansion although it requires only the 8-th order Taylor coefficients. Hence, in our case the Padé approximation reduces the number of required derivatives roughly by a factor of two.

\subsection{Determining the phase boundary and locating the CEP}

The Taylor expansion is limited by the closest singularity in the complex $\mu$-plane. Its distance to the expansion point can be obtained from the convergence radius of the series:

$$
r=\lim _{n \rightarrow \infty} r_{2 n}=\lim _{n \rightarrow \infty}\left|\frac{c_{2 n}}{c_{2 n+2}}\right|^{1 / 2}
$$

If the limiting singularity lies on the real axis the convergence radius corresponds to the location of a critical point. As the limit $n \rightarrow \infty$ cannot be carried out in a numerical study the estimators of the convergence radius at finite $n$ may depend on the considered observable and approach the true convergence radius differently. Therefore we also consider the convergence radius for the quark 


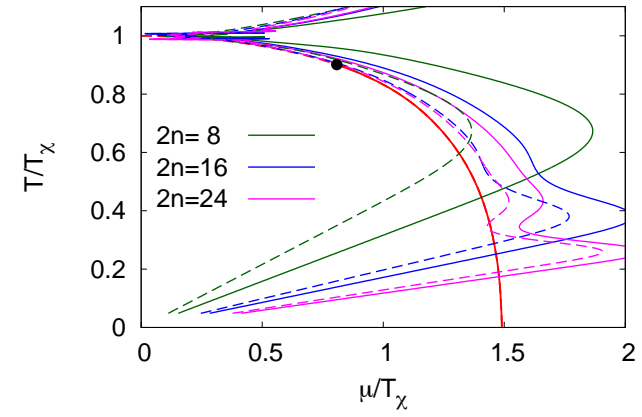

(a)

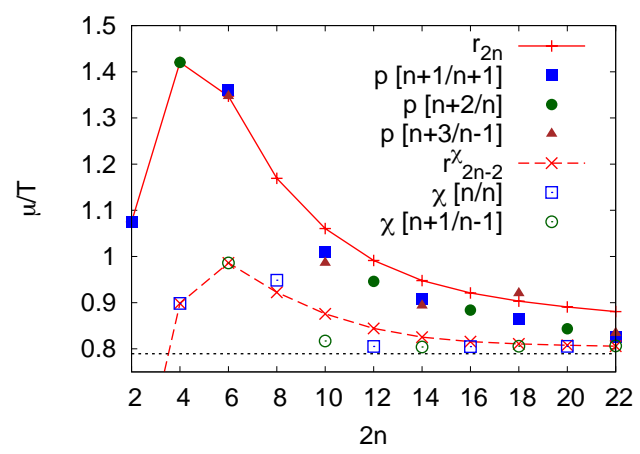

(b)

Figure 3: Left: Estimates for the radius of convergence obtained from $r_{2 n}$ (solid lines) and $r_{2 n-2}^{\chi}$ (dashed lines) for different orders of the Taylor expansion. Also shown is the chiral phase boundary (red line; dashed: crossover, solid: first order). The black dot indicates the CEP. Right: Estimate of the phase boundary at $T=190 \mathrm{MeV} \sim 0.92 T_{\chi}$ with $r_{2 n}$ and $r_{2 n}^{\chi}$ and poles in the Padé approximation of the pressure and quark number susceptibility as a function of the order of the Taylor expansion $2 n$. The used highest coefficient is $c_{2 n+2}$. The dotted horizontal line indicates the phase boundary calculated directly at finite $\mu$.

number susceptibility $r_{2 n}^{\chi}$ which is related to the one of the pressure via

$$
r_{2 n}^{\chi}=\left|\frac{c_{2 n}^{\chi}}{c_{2 n+2}^{\chi}}\right|^{1 / 2}=\left(\frac{(2 n+2)(2 n+1)}{(2 n+3)(2 n+4)}\right)^{1 / 2} r_{2 n+2}
$$

In Fig. 3(a) we show the results for both estimators for three different truncation orders $(2 n=8,16,24)$ in the $(T, \mu)$ phase diagram. Note that the difference in the radii of convergence, estimated from $r_{2 n}$ and $r_{2 n-2}^{\chi}$ is only caused by the prefactor in Eq. (2.6) as the same Taylor coefficients contribute to both estimators. Close to $T_{\chi}$ the oscillations in the Taylor coefficients caused by the imaginary part of the limiting singularity entail oscillations in the radii of convergence and do not allow for a stable estimate of the phase boundary there. For somewhat smaller temperatures the radii of convergence approach the phase boundary from above with increasing truncation order $n$. The observed agreement in the crossover region suggests that the singularity limiting the Taylor expansion is close to the real $\mu$-axis and the small imaginary part is negligible. In particular, this is still valid for the region in the vicinity of the CEP.

The determination of the radius of convergence or the phase boundary with the Pade series is more involved. For the $[N / 2]$ case the Padé approximant has a pole at $x= \pm \sqrt{c_{N} / c_{N+2}}$ which coincides with the estimator $r_{2 N}$, see Eq. (2.5). For a general $[L / M]$ Padé approximant the pole structure is more elaborated. In the general case we use the first pole at real and positive $\mu$ in order to estimate the phase boundary. Since all Taylor coefficients with their corresponding error enter in the Padé approximant the error propagation is more involved here in contrast to the previous discussion where only two error sources enter. In this context, the application of the Padé approximation in lattice simulations is much more involved. However, in the model analysis the coefficients, obtained with the AD technique, exhibit extremely small numerical errors and result in a stable and reliable Padé approximation. In Fig. 3(b) we show for a fixed temperature $T=0.92 T_{\chi}$ the estimates of the phase boundary extracted from Padé approximations for the pressure $(p)$ and 


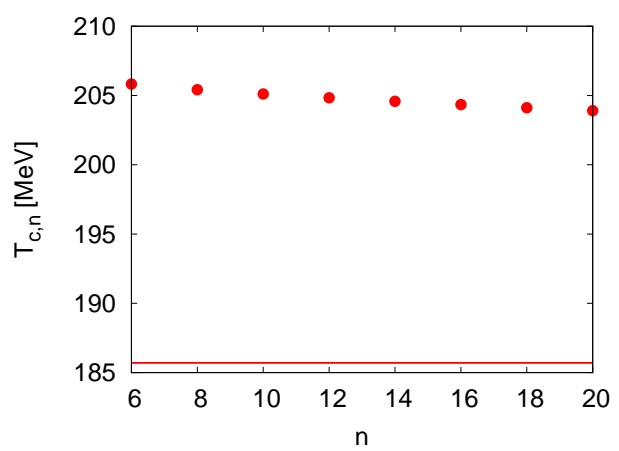

Figure 4: Temperature $T_{c, n}$ at which the Taylor coefficients $c_{n}$ becomes negative for different truncation orders $n$. The solid horizontal line denotes the critical temperature of the CEP.

the quark number susceptibility $(\chi)$ for different truncation orders $n$. The chosen temperature is slightly above the temperature of the CEP in the phase diagram. In addition, the estimates for the radii of convergence $\left(r_{2 n}\right.$ and $\left.r_{2 n}^{\chi}\right)$ and the chiral crossover chemical potential (dashed horizontal line) are also shown. For small truncation orders $2 n \leq 8$ the $[N / 2]$ Padé approximations coincide with the radii of convergence as expected. For $2 n>8$ we observe that the Padé approximation converges faster for the pressure as well as for the susceptibility compared to the radii of convergence of the Taylor series. We conclude that the Padé approximation converges faster at intermediate truncation orders in particular for the quark number susceptibility. The estimate of the phase boundary from the Padé approximation becomes comparable to the one obtained from the Taylor expansion coefficients only at significantly larger truncation order. For example, in Fig. 3(b) the distance of the Padé estimate to the horizontal line at $2 n=12$ is achieved with Taylor coefficients only for $2 n \geq 20$. However, the lower truncation order in the Padé approximation induces a more involved error propagation which might hamper lattice simulations but not calculations with the AD-techniques.

From the discussion given so far and from Fig. 3(b) one may conclude that at fixed temperature the radius of convergence can be estimated with an accuracy of about $15 \%-20 \%$ from a $\mathscr{O}\left(\mu^{12}\right)$ series expansion. This is an acceptable uncertainty in view of the difficulties in current QCD calculations at non-zero chemical potential. When $T_{c}$ is known the CEP could be determine from

$$
\mu_{c}=r\left(T_{c}\right)=\lim _{n \rightarrow \infty} r_{n}\left(T_{c}\right)
$$

However, the determination of $T_{c}$ is still a non-trivial task. A criterion is needed, that allows to estimate for a given expansion order the temperature regime where all expansion coefficients may stay positive. In this case the singularity limiting the convergence is located on the real axis in the complex $\mu$-plane and hence corresponds to a second-order phase transition [13]. In Fig. 4 we plot the temperature $T_{c, n}$ where the Taylor coefficient $c_{n}(T)$ becomes negative. This yields an upper bound for the critical temperature. Of course, in the limit $n \rightarrow \infty$ the temperature series should approach the critical temperature of the CEP, i.e.,

$$
T_{c}=\lim _{n \rightarrow \infty} T_{c, n}
$$


However, we found in our model analysis only a very slow convergence of the estimators for the critical temperature of the CEP. Even at the 20-th truncation order there is still a temperature difference of the order of $20 \mathrm{MeV}$ which is why we do not apply Eq. (2.7).

\section{Summary and Conclusion}

In this talk we discussed convergence properties of the Taylor expansion towards finite density QCD in a three flavor PQM model framework. A newly developed differentiation technique allowed for the calculation of high order Taylor coefficients with high precision. With a Padé resummation the number of required Taylor coefficients can be reduced by a factor of two to obtain a similar convergence radius. For temperatures above the critical endpoint a good agreement of the radius of convergence with the phase boundary is found, in particular for the estimator of the quark number susceptibility. With a 12-th order Padé approximation a satisfying estimate of the phase boundary could be achieved. However, a determination of the critical temperature including the location of the CEP is hampered by the slow convergence of the estimators $T_{c, n}$.

Support by the BMBF Grant 06BI9001 and by the Helmholtz-University Young Investigator Grant No. VH-NG-332 is acknowledged.

\section{References}

[1] L. Levkova, these proceedings, PoS LAT2011, (2011).

[2] O. Philipsen, PoS LAT2005, 016 (2006); C. Schmidt, PoS LAT2006, 021 (2006); P. de Forcrand, PoS LAT2009, 010 (2009).

[3] C. R. Allton et al. Phys. Rev. D66, 074507 (2002); Phys. Rev. D68, 014507 (2003); Phys. Rev. D71, 054508 (2005). R. V. Gavai and S. Gupta, Phys. Rev. D68, 034506 (2003); Phys. Rev. D71, 114014 (2005); Phys. Rev. D78, 114503 (2008).

[4] F. Karsch, B.-J. Schaefer, M. Wagner, and J. Wambach, Phys.Lett. B698, 256 (2011).

[5] C. W. Bernard et al. (MILC Collaboration), Phys.Rev. D55, 6861 (1997); F. Karsch, E. Laermann, and A. Peikert, Phys.Lett. B478, 447 (2000); A. Ali Khan et al. (CP-PACS), Phys. Rev. D64, 074510 (2001).

[6] B.-J. Schaefer, M. Wagner, and J. Wambach, Phys. Rev. D81, 074013 (2010).

[7] S. Rößner, C. Ratti, and W. Weise, Phys. Rev. D75, 034007 (2007).

[8] B.-J. Schaefer and M. Wagner, Phys. Rev. D79, 014018 (2009).

[9] B.-J. Schaefer et al., Phys. Rev. D76, 074023 (2007); T.K. Herbst et al., Phys. Lett. B696 , 58 (2011).

[10] M. Wagner, A. Walther, and B.-J. Schaefer, Comp. Phys. Commun. 181, 756 (2010).

[11] B.-J. Schaefer, M. Wagner, and J. Wambach, PoS CPOD2009, 017 (2009); J. Wambach, B.-J. Schaefer, and M. Wagner, Acta Phys. Pol. B Proc. Suppl. 3, 691 (2010).

[12] C. Schmidt, PoS CPOD2009, 024 (2009).

[13] M. A. Stephanov, Phys. Rev. D73, 094508(2006); C.-N. Yang and T. Lee, Phys. Rev. 87, 404 (1952).

[14] B.-J. Schaefer and M. Wagner, in preparation (2011). 